\title{
Scaling of the Mammalian Brain: the Maternal Energy Hypothesis
}

\section{Robert D. Martin}

\begin{abstract}
Mammalian brain sizes have been linked to specific behavioral or physiological features because of simple scaling correlations. Examination of the correlation network for body size, brain size, basal metabolic rate, and gestation period indicates that the primary link is between maternal metabolic capacity and the developing brain of the offspring.
\end{abstract}

\section{$\mathbf{T}$}

he concept of scaling is now well established, and allometric analysis has become a standard tool for quantitative comparison of features between organisms of differing body size. This technique has been applied with great success in the field of comparative physiology, notably to respiratory physiology of mammals and of the avian egg, and the major findings have been succinctly reviewed by Knut SchmidtNielsen (14). Comparative physiologists have repeatedly used scaling analyses to explore functional relationships, but in other fields the main objective has been to identify general scaling trends to exclude body size effects from comparisons between species. Thus various studies of comparative morphology, reproductive biology, and behavioral ecology have focused on distinctions between taxonomic groups and associated evolutionary relationships, without much concern for underlying functional relationships.

Many authors trace the origins of the modern concept of allometric scaling back to Julian Huxley's pioneering treatise on quantitative aspects of growth (6), but the first significant application of the concept of allometric scaling was in fact made in studies of mammalian brain size at the turn of the century by [ugène Dubois (3), the discoverer of Homo erectus. Dubois introduced and applied the standard allometric formula that is now almost universally used in scaling studies. Scaling relationships broadly conform to the power function $Y=k X^{\alpha}$ ( $\alpha$ being the scaling exponent and $k$ the scaling coeffi-

R. D. Martin is Director of the Anthropological Institute and Museum of the University of Zurich-Irchel, Winterthurerstrasse 190, CH-8057 Zurich, Switzerland. cient), and logarithmic transformation yields the linear equation $\log Y-\alpha \cdot \log X+\log k$, which is more amenable to straightforward statistical treatment (Fig. 1).

Scaling analyses based on the concepts illustrated in Fig. 1 have various applications. In addition to recognition of general scaling principles and identification of outliers and grades, empirically determined scaling relationships can be used to lest hypotheses, lo make prediclions, and to explore potential functional relationships. It must be emphasized, however, that this latter application requires great caution. Many scaling analyses are confined to the relationship between a single variable and body weight, and it is vital to recognize that many such relationships exist, such that a correlation in any one analysis does not necessarily indicate a causal connection. Functional relationships can only be inferred with any confidence following comprehensive scaling analyses of an entire set of interconnected features.

\section{Scaling of basal metabolism}

Perhaps the most widely recognized example of allometric scaling in comparative physiology is the relationship between basal metabolic rate and body size in placental mammals $(1,9)$. It is now widely (if not universally) accepted that the interspecific scaling exponent for this relationship is 0.75 , and analyses of large data samples provide strong empirical support (Fig. $2 A$ ). Recognition of this exponent value was particularly advocated by Max Kleiber (9), and the empirical scaling principle is now commonly referred to as Kleiber's law. The relationship is relatively tight; although individual species deviate from the
"Scaling relationships broadly conform to the power function $Y=k X^{\alpha} \ldots . "$ 


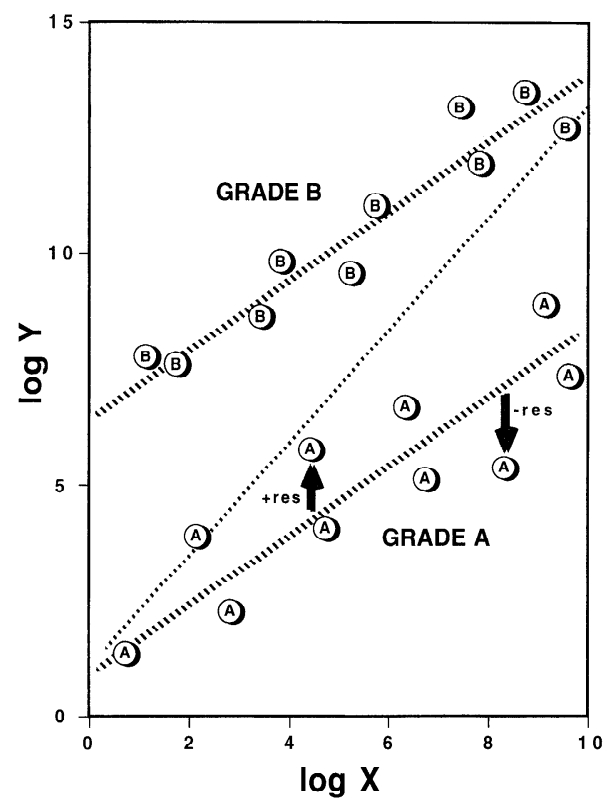

FIGURE 1. Basic illustration of scaling relationships in comparative biology. Data for body weight $(X)$ and a chosen biological variable $(Y)$ are presented in a logarithmic bivariate plot. A best-fit line determined for a given group of species (e.g., points marked $A$ ) indicates overall scaling trend; exponent $\alpha$ in the allometric equation is directly identifiable as slope of the line, and intercept is $\log k$. Within a given group, deviations of individual species from best-fit line (residual values) indicate specific positive or negative adaptations (+res, - - res; shaded areas show typical ranges of such deviations). Residual values can be used as an index of relative size of $Y$ after taking scaling effect of body size $(X)$ into account. The 2 groups of species $A$ and $B$ exhibit separate scaling relationships defined as grades (indicated by hatched best-fit lines). In this example, slope indicating scaling exponent $(\alpha=0.75)$ is same in both cases, but intercept $(-\log k$ ) differs between the 2 groups of species. If an overall best-fit line is determined for all species taken together (thin dotted line), a line with a markedly greater slope of 1.22 is obtained. (All lines are reduced major axes.)

best-fit line, such deviations are quite limited. This is reflected in the high value of the correlation coefficient $(r=0.98$ for the data in Fig. $2 A)$. The extremes are represented by species that have approximately three times the value predicted by the best-fit line and those that have aboul one-third of the predicted value. Although the exponent value of 0.75 for scaling of basal mctabolic rate remains empirical, since there is still no widely accepted theoretical explanation, the relationship has been broadly and successfully applied (14).

Despite the restricted range of deviation from the general scaling trend for basal metabolic rate in mammals, various grades (Fig. 1) and individual outliers can be recognized. It is, for instance, well known that (relative to body size) marsupials tend to have lower basal metabolic rates than placental mammals $(\sim 25 \%$ on average). Among primates, prosimians (lemurs, lorises, and tarsiers) generally have relatively low basal metabolic rates, whereas simians (monkeys, apes, and humans) typically lie quile close to the line for Kleiber's law. It has also been noted for mammals generally that leaf-eating species tend to have lower basal metabolic rates than fruit eaters and that insectivorous bats typically have lower basal metabolic rates than fruit-eating bats (10).

\section{Scaling of mammalian brain size}

Ever since the early work of Dubois, the scaling of brain size relative to body size in mammals has remained one of the prime examples of empirical scaling relationships. An extensive literature was reviewed in 1973 by Harry Jerison (7). At that time, the dominant interpretation was that the scaling exponent for this relationship has the value 0.67 , and a possible functional explanation was based on the surfaceto-volume ratio. For a given body shape, body surface area increases according to the square of body length, whereas body weight increases according to the cube. Accordingly, the exponent value for scaling of a surface-to-volume relationship will be $2 / 3$ or 0.67 . One simplistic explanation for a scaling exponent value of $(0.67$ was based on the argument that many receptors and effectors are distributed over external or internal body surfaces, such that the brain connected with them might be expected to scale in relation to body surface area rather than its volume or weight. Of course, much of the brain is not connected in this simple manner with receptors and effectors.

In fact, surface-volume scaling was subsequently ruled out on empirical grounds because independent analyses of large data sets indicated a scaling exponent significantly greater than 0.67 and in fact quite close to 0.75 (10) (Fig. 2B). There is an interesting parallel here in that it was originally believed that basal metabolic rate scales with an exponent value of 0.67 to body weight, fitting the expectation that heat is generated or required by a body according to its volume, whereas gain or loss of heat from or to the external environment would be dependent on body surface area. It was eventually shown convincingly by Brody and Kleiber, with the "mouse-to-elephant curve," that the empirical exponent value for the scaling of basal metabolic rate is 0.75 , not 0.67 . Empirical scaling cxponents for basal metabolism and for brain size are hence both close to 0.75 . In other words, the relationship between brain size and basal metabolic rate is directly proportional or isometric $(\alpha \sim 1)$, suggesting a possible link between brain size and metabolic turnover. 

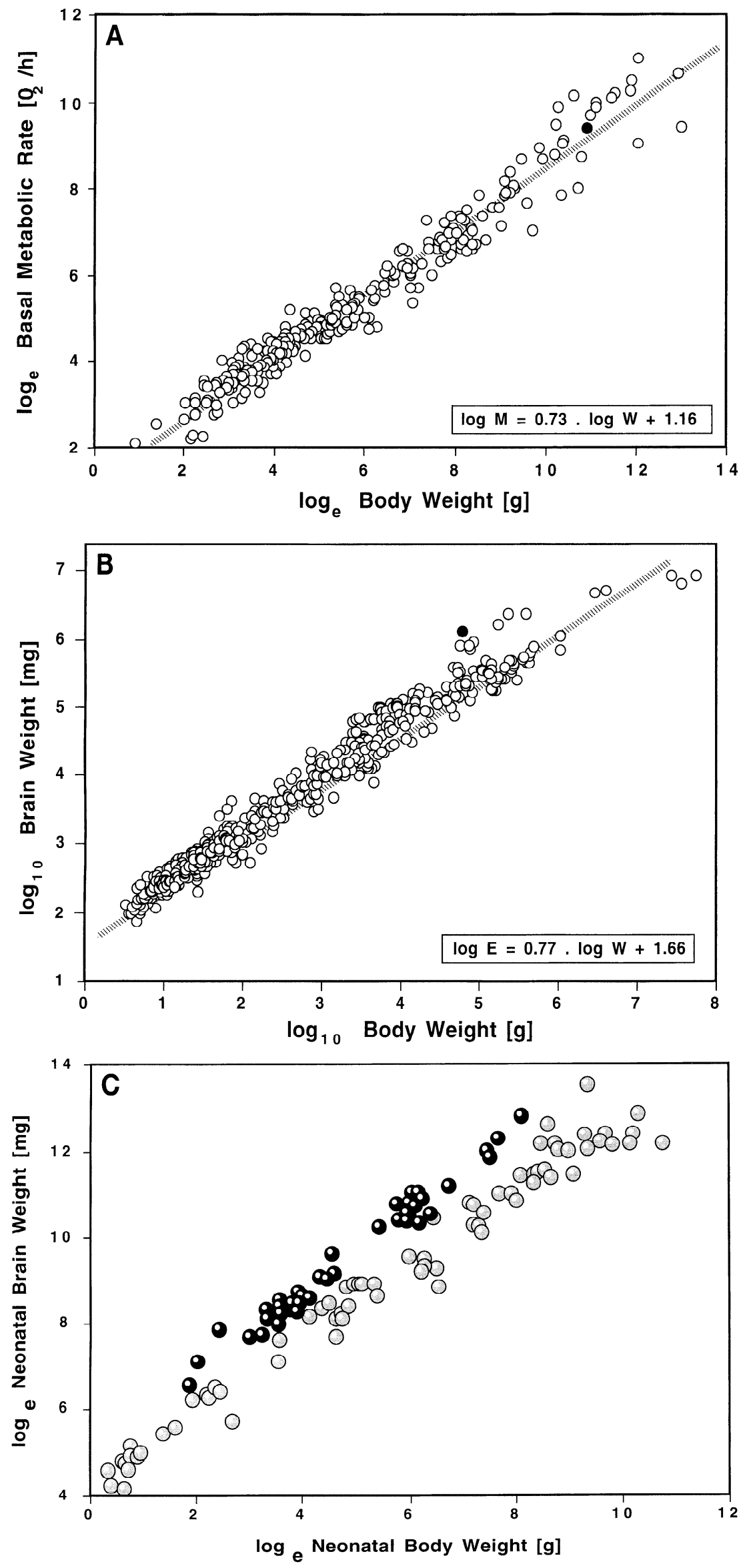

FIGURE 2. A: scaling of basic metabolic rate $(\mathcal{M})$ against body weight $(W)$ in a large sample of placental mammal species $[n=269$; data from McNab (11)]. Empirical scaling formula is $\log _{\mathrm{e}} M=0.73$ $\log _{\mathrm{e}} W+1.16(r-0.98)$. Solid circle is Homo sapiens. $B$ : scaling of adult brain weight $(E)$ against adult body weight $(W)$ in a large sample of placental mammal species ( $n=477$; author's data set). Empirical scaling formula is $\log _{10} E=0.77 \log _{10} W+1.66$ $(r=0.98)$. Solid circle is Homo sapiens. C: scaling of nconatal brain weight $\left(E_{n}\right)$ against neonatal body weight $\left(W_{\mathrm{n}}\right)$ for a sample of placental mammals ( $n=117$; author's data set). Points for primates (black circles; $n=43$ ) consistently lie above points for nonprimates (stippled circles; $n=$ 74). This is a clear-cut grade relationship, reflecting the fact that in primates brain represents a larger proportion of fetal body mass throughout development than in nonprimates. 
". . . changes that took place following their divergence from that common ancestor."

\section{Problem of statistical independence of species}

The empirical value of 0.75 for the exponent for scaling of brain size to body size in placental mammals, based on analyses of large samples (10), was subsequently questioned from a new direction. The challenge was based on the statistical argument that species in such a comparative scaling analysis may not represent independent data points $(4,5)$. The reason for this is that individual specics are, of course, related to one another to varying degrees within the evolutionary tree of mammals. It is therefore theoretically possible that values for closely related species may introduce a statistical bias into the analysis. Hence, the use of statistical treatments, such as standard line-fitting procedures, that require independence between individual data points could be inappropriate.

One approach to counter the potential problem of bias arising from differential degrees of relationship between species is that of phylogenetic contrasts $(4,5)$. This is based on the following reasoning: if species $A$ and species $B$ arc derived from a common ancestor, it may be the case that the values they exhibit for the two variables of interest $\left(X_{A}, Y_{A}\right.$ and $X_{B^{\prime}} Y_{B^{\prime}}$, respectively) are both heavily influenced by the values present in the common ancestor. On the other hand, the differences in the values ("contrasts") between $A$ and $B$ reflect changes that took place following their divergence from that common ancestor. Instead of examining the scaling of the raw values, therefore, one can examine the scaling of the contrasts between them in the belief that this will eliminate the problem of statistical interdependence between data points. For logarithmic values, the relationships involved are in principle quitc simple, as is shown by the following equations for species $A$ and $B$ (assuming perfect scaling)

$$
\begin{aligned}
& \log Y_{A}=\alpha \cdot \log X_{A}+\log k \\
& \log Y_{B}=\alpha \cdot \log X_{B}+\log k
\end{aligned}
$$

thus

$$
\log Y_{A}-\log Y_{B}=\alpha\left(\log X_{A}-\log X_{B}\right)
$$

Accordingly, a plot of contrasts in $Y$ values (e.g., $\left.\log Y_{A}-\log Y_{B}\right)$ against the corresponding contrasts in $X$ values (e.g., $\log X_{A}-\log X_{B}$ ) for a given set of species should yield a line of slope $\alpha$ passing through the origin. Following this rationale, Harvey and Pagel (5) confirmed that the scaling exponent for basal metabolic rate in placental mammals is close to 0.75. However, they also conducled an analysis of mammalian brain and body weights and determined a scaling exponent value of 0.69 . This is closer to the value of 0.67 expected from surface scaling than to the value of 0.75 expected from a link with metabolic scaling and throws doubt on the suggestion that there is some connection between brain size and basal metabolism.

There are, however, numerous problems with the use of contrast values for determining empirical scaling relationships. In the first place, the equations given above apply only if the two taxa compared belong to the same grade and hence have the same value of the scaling coefficient $k$ (Fig. 1). If a grade shift separates two species in a comparison, it will distort the contrast value. Second, contrasts determined between closely related taxa will be particularly problematic because close similarity in body size will result in very small contrast values that are particularly prone to error. Errors in contrast values for closely related taxa combined with the effects of multiple grade shifts might therefore invalidate the results of contrast analysis. Last but not least, the slope of an empirical scaling relationship is influenced mainly by values at the upper and lower extremes of the distribution, such that phylogenetic relatedness should not be expected to have much effect on the overall relationship determined. One way of countering the potential problem of statistical interdependence between closely related species while taking these objections into account is to conduct a simplified analysis using overall average values for mammalian orders. Reanalysis of McNab's data (11) for basal metabolic rate (Fig. 2A) using averaged values for 16 orders of mammals yields a scaling exponent of $0.76(r=0.97)$. For the 15 contrast values determined from these ordinal averages using the program CAIC (12), a line passing though the origin yields a scaling exponent of 0.82. In this case, considerable scatter of the points in the contrast plot probably reflects grade differences between mammalian orders in basal metabolic rate, and this could have led to some distortion of the inferred exponent value. A parallel reanalysis of the data for adult brain size (Fig. 2B) using averaged values for 14 orders of mammals yields a scaling exponent of $0.77(r=$ 0.99 ). For the 13 contrast values derived from these ordinal averages, a line passing though the origin yields an almost identical scaling exponent of 0.76 , and there is very little scatter of points. Overall, these results show that the scaling exponents for both basal metabolic rate and adult brain size are consistently higher than 0.67 and generally close to the value of 0.75 . Thus the possibility of a link between basal metabolic rate and brain size remains. 


\section{Scaling of the brain in primates}

Having established a reliable empirical scaling relationship between brain size and body size for a large sample of placental mammal species, one can turn to interpretation of the residual values of individual species and groups of species. First, it should be noted that the range of residual values exceeds that found with basal metabolic rate, extending from species that have approximately five times the brain size predicted by the best-fit line to those that have about one-fifth of the predicted value. Second, it has been widely claimed that adult primates have bigger brains than other adult mammals, but there is in fact no foundation for this. It is certainly not true in terms of absolute brain size, since an elephant's brain, for example, is four times bigger than that of a human, and it is not even true with respect to relative brain size. Examination of the residual values for various orders of placental mammals shows that there is considerable overlap between primates and nonprimates and that a few primate species actually lie close to or even below the average condition for mammals. While it is true that the average residual value for primates is greater than the average value for other mammalian orders, there is no discrete grade shift between primates and other mammals. Indeed, although humans do have the largest relative brain sizes in the sample, the next largest relative brain sizes are not found in other primates at all, but in dolphins (members of the order Cetacea).

Nevertheless, examination of a different aspect of the relationship between brain size and body size reveals a systematic grade distinction between primates and other mammals. This distinction lies in the growth of the brain relative to the growth of the rest of the body throughout gestation. As was originally shown by George Sacher (13), the brain of a primate fetus at any stage of development constitutes a markedly larger proportion of total fetal weight than in other mammals ( $\sim 12 \%$ as compared with $6 \%$ ). Because this difference is consistently present throughout gestation, it is still observable in neonates (10). Hence, a plot of neonatal brain weight against neonatal body weight (for which a much bigger sample is available) also reveals this clear systematic difference between primates and other mammals (Fig. 2C). Hence, primates as a group do have greater relative brain sizes than other mammals at birth, but because of differential postnatal changes in different mammals, this difference is no longer clear in comparisons among adults. This finding is important because it shows that the development of the brain, and not just its ultimate size in the adult, may be crucial.

\section{Explanations of relative brain size}

Many attempts have been made to explain differences in relative brain size among primates, with special emphasis on humans. Various links to specific behavioral and/or ecological variables have been suggested, usually on the basis of single correlations. One correlation that was noted early on for primates is that leaf-eating species generally tend to have smaller brains than fruit-eating species (2). A possible explanation of this correlation is that a greater central nervous processing capacity is required to locate fruits than leaves in tropical forests inhabited by primates. Links have also been suggested between relative size of the brain or parts thereof and complexity of social organization. Most recently, it has been proposed that there is a trade-off between the size of the brain and the size of the digestive system, which could explain why leaf-eating primates have smaller brains than fruit-eating primates (1). There is, however, an alternative explanation for all of these findings. Leaf-eating primates generally have lower basal metabolic rates than fruit-eating primates (10), and this may constrain their energy expenditure in various directions. The low metabolic rates of leaf-eaters may account not only for their smaller brains but also for their smaller social groups, and it is obvious that digestion of leaves will require a relatively larger digestive tract. Indeed, support for a metabolic link is provided by the observation that fruit-eating bats have relatively larger brains than insect-eating bats. As noted above, fruiteating bats also have relatively higher metabolic rates than insect-eating bats (10). In this case, there are no apparent correlations either with social complexity or with the relative size of the digestive tract, so the explanations suggested for primates have no general validity. Indeed, comparative studies show that insectivorous mammals typically have relatively smaller digestive tracts than fruit-eating mammals, so the hypothesis that there is a trade-off between the brain and the gut in primates (1) receives no support from bats.

Even if it is accepted that the primary link is between basal metabolism and brain size, different interpretations are possible. The observed correlation could be explained either by dependency of basal metabolism on adult brain size or by dependency of adult brain size on basal metabolism. (Both possibilities have, in fact, been suggested in the literature.) In fact, how-
". . the brain of a primate fetus ... constitutes a markedly larger proportion of total fetal weight...." 


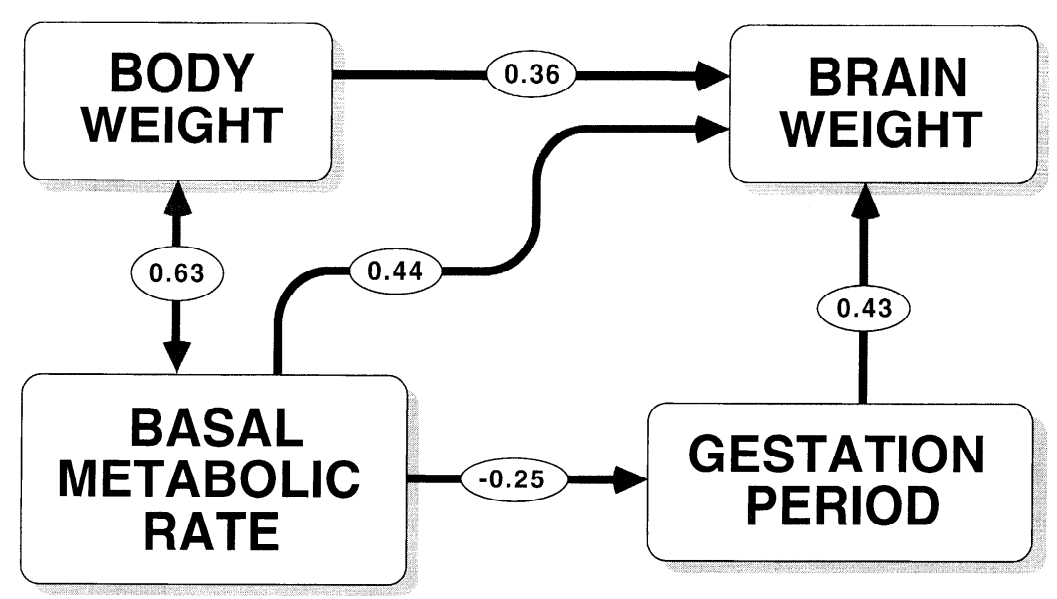

FIGURE 3. Flow diagram showing partial correlations between body weight, brain weight, basal metabolic rate, and gestation period with possible directions of effects. Note that there is no remaining association between body weight and gestation period and that partial correlation between basal metabolic rate and gestation is negative.

ever, a direct link in either direction between basal metabolism and brain size in the adult can be ruled oul for a number or reasons. First, it has already been noted that residuals for brain size show a fivefold range of variation on either side of the best-fit line, whereas residuals for basal metabolism show only a threefold range of variation. This indicates that a significant amount of variation in adult brain size is not explained by variation in basal metabolic rate. This is confirmed by a direct plot of adult brain size against basal metabolic rate, which shows considerable scatter. Second, one cannot account for the very large size of the human brain (Fig. 2B) on this basis, since humans lie more or less on the best-fit line for scaling of basal metabolic rate to body weight in mammals (Fig. $2 A)$. Hence, if there is a connection between basal metabolic rate and adult brain size, it must be indirect.

\section{Maternal energy hypothesis}

Various lines of evidence, including the grade distinction for neonatal primates presented in Fig. $2 C$, indicate that the development of the brain is of particular significance in scaling relationships, and this led the author to suggest that there is a link between the basal metabolic rate of the mother (an indicator of her energy turnover) and the brain size of her developing offspring. The brain is unusual among body organs in that most of its growth is completed at a very early stage, and hence resources provided by the mother during pregnancy and lactation support the main phase of brain development. If the primary link is between the mother's metabolic capacity and the developing brain of her offspring, this would allow other variables to influence ultimate adult brain size. Both the length of gestation and the period of postnatal growth supported by lactation could exert modifying effects, thus explaining why there is no tight scaling relationship between the brain size of an adult and its own basal metabolic rate.

The dense network of individual correlations involved can be tackled by calculating partial correlations between pairs of variables, having excluded the effects of all others. With data on body weight, brain weight, basal metabolic rate, and gestation period taken into account for a sample of 53 placental mammal species, standard pairwise comparison indicales strong and significant correlations between all variables. Partial correlations $\left(r_{\mathrm{p}}\right)$, on the other hand, reveal that additional variables exert a confounding influence on the relationship between any two variables. Indeed, the original correlation between gestation period and body weight virtually disappears when the effects of basal metabolic rate and brain weight are taken into account (reduction from $r=0.71$ to $r_{\mathrm{p}}=0.07$ ), while the original positive correlation between gestation and basal metabolic rate is transformed into a negative correlation (conversion from $r=0.69$ to $\left.r_{\mathrm{p}}=-0.25\right)$. The results of partial correlation analysis can be presented in the form of a flow diagram (Fig. 3), showing the remaining associations and the possible direction of effects. From this, it can be seen that (as expected) a strong partial correlation remains between basal metabolic rate and body weight but that the partial correlation between body weight and brain weight is considerably reduced. Both basal metabolic rate and gestation period show stronger partial correlations with brain weight than does body weight, as would be expected from the hypothesis that ultimate adult brain size is dependent primarily on the mother's basal meta- 
bolic rate mediated (among other things) by the gestation period. The negative partial correlation between basal metabolic rate and gestation period is also compatible with the hypothesis. If the mother has a low basal metabolic rate, this is likely to constrain the rate of fetal growth, and one way of offsetting this is to extend the gestation period. Hence (other things being equal), species with relatively low basal metabolism might be expected to have relatively long gestation periods.

One factor that might be expected to influence the development of the fetal brain, in addition to the availability of maternal metabolic resources and the length of the gestation period, is the efficiency of transfer of resources across the placenta. Interestingly, however, the structure of the placenta does not seem to be a limiting factor for the development of the mammalian brain. While it is true that humans, with the largest relative brain size among mammals, have a highly invasive hemochorial placenta (presumed to maximize transfer between mother and fetus), the next largest relative brain size is found in dolphins, which have a noninvasive epitheliochorial placenta, widely regarded as being relatively "inefficient."

Independent evidence for the existence of a special relationship between the mother and the development of her offspring's brain has recently been provided by work on genomic imprinting by Barry Keverne and colleagues (8). Genomic imprinting leads to differential expression of maternal or paternal genes during development in mammals. With the use of a transgenic procedure, mouse embryos were produced in which either the paternal or the maternal genomc had been duplicated at the expense of that of the other parent. Cells with a duplicated paternal genome contributed substantially to basal parts of the brain (hypothalamic structures), while those with a duplicated maternal genome contributed substantially to more advanced parts of the brain (cortex, striatum, and hippocampus). Furthermore, growth of the brain overall was promoted by a duplicated maternal genome but retarded by a duplicated paternal genome. These findings clearly indicate a special role for the mother in the evolution of mammalian brain size.

\section{Implications for human brain size}

The maternal energy hypothesis has a number of implications for interpretation of relative brain size. In the first place, various correlations between brain size and behavioral or ecological variables may be a secondary consequence of a primary link between the basal metabolic rate of a mother and the developing brain of her offspring. Second, there may be no very tight relationship between relative brain size and specific behavioral capacities. The latter may be far more dependent on the internal wiring of the brain rather than its overall size. While it is undoubtedly true that large brains are generally superior to small brains, no convincing case has been made for the proposal that any particular feature of behavior (e.g., feeding ecology, complexity of social organization) has exerted a specific selection pressure favoring an increase in brain size. Instead, it seems more likely that an increase in brain size is advantageous in a diffuse fashion, as is witnessed by the fossil evidence that relative brain size has increased in all mammalian lineages over time (albeit at different rates). The maternal energy hypothesis proposes that all mammals have the largest brains that are compatible with the metabolic resources available to their mothers during gestation and laclation, and it should therefore not be expected that relative brain size will be specifically linked to a given behavioral function.

This hypothesis has important implications for the discussion of brain size in humans. As for primates generally, various specific links have been suggested between brain size and individual behavioral features such as social complexity, along with certain special human features such as advanced tool-making capacity and language. However, when the effects of confounding variables such as body size and socioeconomic status are excluded, no correlation is found between IQ and brain size among modern humans. Until some behavioral advantage of increased brain size per se has been demonstrated, there is no basis for arguing that specific selection pressures have favored the development of a very large brain in humans.

In fact, there is one puzzling feature of modern human brain size that is quite incompatible with any proposed link between brain size and specific behavioral capacities. It is widely recognized that the Neanderthals had a larger brain than modern humans, but it has now emerged that anatomically modern humans also had larger brains some 30,000 years ago and that our brain size seems to have declined progressively since then (Fig. 4). It has been suggested that this is simply a consequence of a progressive decline in body size, but there is no good skeletal evidence to confirm this. In any case, human brain size has been declining in size over the very period (the last 30,000 years), during which our most impressive cultural achievements have become apparent. This apparent paradox might
"... all mammals have the largest brains that are compatible with the metabolic resources available to their mothers ...." 


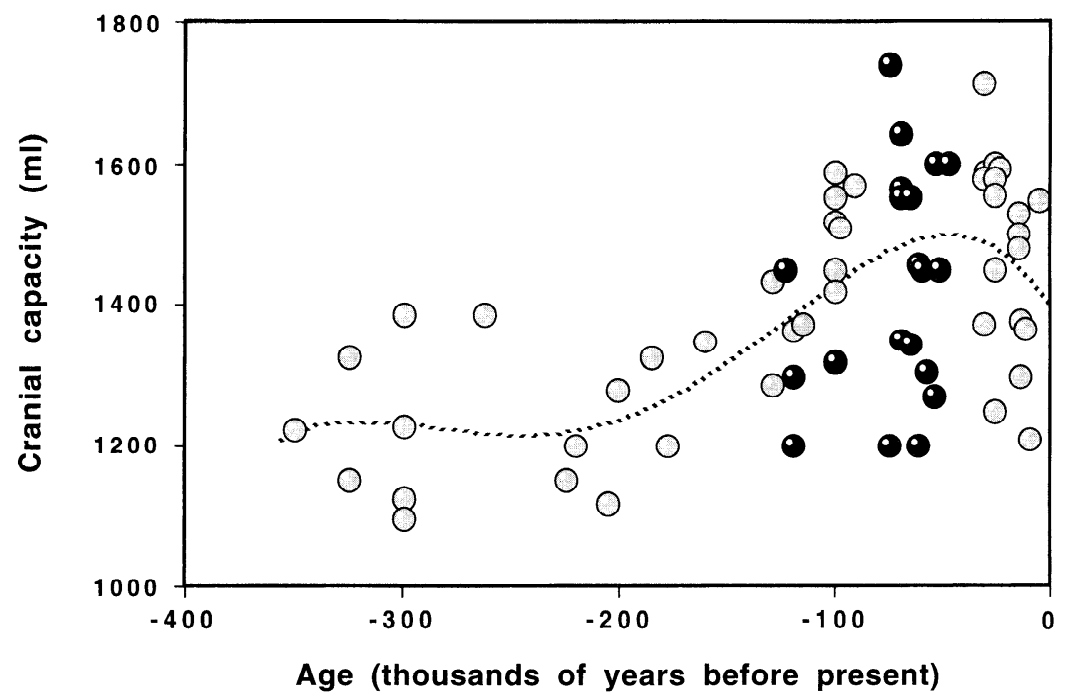

FIGURE 4. Changes in hominid cranial capacity over the past 400,000 years. A general increase over period between 200,000 and 30,000 years ago has been followed by a progressive decline toward level found in modern Homo sapiens. Both Neanderthals (solid circles) and earlier anatomically modern humans had larger brains than modern humans. [Data from Stanyon et al. (15).]

be resolved if it is accepted that there is no direct link between behavioral capacities and brain size in mammals. Indeed, the maternal energy hypothesis would indicate that the explanation for the progressive decline in human brain size should in fact be sought in changes in the allocation of maternal resources to fetal and postnatal development.

\section{References}

1. Aiello, L. C., and P. Wheeler. The expensive tissue hypothesis: the brain and the digestive system in human and primate evolution. Curr. Anthropol. 36: 199-221, 1995.

2. Clutton-Brock, T. H., and P. H. Harvey. Primates, brains and ecology. J. Zool. Lond. 190: 309-323, 1980.

3. Dubois, E. On the relation between the quantity of brain and the size of the body in vertebrates. Proc. K. Ned. Akad. Wet. 16: 647-667, 1913.

4. Felsenstein, J. Phylogenies and the comparative method. Am. Nat. 125: 1-15, 1985.

5. Harvey, P. H., and M. D. Pagel. The Comparative Method in Evolutionary Biology. Oxford, UK: Oxford Univ. Press, 1991.

6. Huxley, J. S. Problems of Relative Growth. London: Methuen, 1932.
7. Jerison, H. J. Evolution of the Brain and Intelligence. New York: Academic, 1973.

8. Keverne, E. B., R. Fundele, M. Narasimha, S. C. Barton, and M. A. Surani. Genomic imprinting and the differential roles of parental genomes in brain development. Dev. Brain Res. 92: 91-100, 1996.

9. Kleiber, M. The Fire of Life: An Introduction to Animal Energetics. New York: Wiley, 1961.

10. Martin, R. D. Human Brain Evolution in an Ecological Context (52nd James Arthur Lecture on the Evolution of the Human Brain). New York: Am. Museum Natural History, 1983.

11. McNab, B. K. Basal metabolic rate and phylogeny. Funct. Ecol. 1: 159-167, 1987.

12. Purvis, A., and A. Rambaut. Comparative analysis by independent contrasts (CAIC): an Apple Macintosh application for analysing comparative data. Comput. Appl. Biosci. 11: 247-251, 1995.

13. Sacher, G. A. The role of brain maturation in the evolution of the primates. In: Primate Brain Evolution, edited by E. Armstrong and D. Falk. New York: Plenum, 1982, p. 97-112.

14. Schmidt-Nielsen, K. Scaling: Why Is Animal Size So Important? Cambridge, UK: Cambridge Univ. Press, 1984.

15. Stanyon, R., S. Consigliere, and M. A. Morescalchi. Cranial capacity in hominid evolution. Hum. Evol. 8: 205-216, 1993.

\section{In Forthcoming Issue}

Homeostasis, Humpty Dumpty, and Integrative Biology Stanley G. Schultz

One Hundred Years of Starling's Hypothesis Charles C. Michel

Chemotropic Factors Direct Regenerating Axons Damien P. Kuffler 